\title{
Writing direction affects how people map space onto time
}

\author{
Benjamin K. Bergen ${ }^{1}$ and Ting Ting Chan Lau ${ }^{2}$ \\ 1 Department of Cognitive Science, University of California San Diego, San Diego, CA, USA \\ ${ }^{2}$ Department of Linguistics, University of Hawaii, Honolulu, HI, USA
}

\author{
Edited by: \\ Asifa Majid, Max Planck Institute for \\ Psycholinguistics, Netherlands \\ Reviewed by: \\ Ayse K. Uskul, University of Essex, \\ UK \\ Yulia Chentsova Dutton, Georgetown \\ University, USA \\ *Correspondence: \\ Benjamin K. Bergen, Department of \\ Cognitive Science, University of \\ California San Diego, 9500 Gilman \\ Drive, San Diego, CA, USA. \\ e-mail:bkbergen@cogsci.ucsd.edu
}

What determines which spatial axis people use to represent time? We investigate effects of writing direction. English, like Mandarin Chinese in mainland China, is written left to right and then top to bottom. But in Taiwan, characters are written predominantly top to bottom and then right to left. Because being a fluent reader-writer entails thousands of hours of experience with eye and hand movement in the direction dictated by one's writing system, it could be that writing system direction affects the axis used to represent time in terms of space. In a behavioral experiment, we had native speakers of English, Mandarin Chinese from mainland China, and Mandarin Chinese from Taiwan place sets of cards in temporal order. These cards depicted stages of development of plants and animals, for instance: tadpole, froglet, frog. Results showed that English speakers always represented time as moving from left to right (LR). Mainland Chinese participants trended in the same direction, but a small portion laid the cards out from top to bottom. Taiwanese participants were just as likely to depict time as moving from LR as from top to bottom, with a large minority depicting it as moving from right to left. Native writing system affects how people represent time spatially.

Keywords: time, space, writing direction, English, Mandarin Chinese

\section{INTRODUCTION}

\section{THERE ARE DIFFERENT SPATIAL CONSTRUALS OF TIME}

Despite its pervasive presence in our thought and speech, time has no material substance. It is not directly perceivable through the senses - it does not look like anything or sound like anything. Nor is it something you can have direct motor knowledge about because it is not something you do. Yet across languages and cultures, people have converged upon globally similar solutions for thinking and talking about this most abstract of concepts. One such solution is to talk and think about time in terms of space.

It is been long noted that we use language about space to describe time (for instance, Clark, 1973; Traugott, 1978; Lakoff and Johnson, 1980). English often is not explicit about the direction of the metaphorical motion it ascribes to time (The days are flying $b y$ ), but when it is, the experiencer and time are often interpreted as moving past each other on some horizontal axis (Christmas is still ahead of us; The school year is behind us).

What's more, these linguistic patterns also appear to reflect conceptual relations between time and space. Evidence comes from behavioral experiments. Having people perceive particular spatial configurations can affect their subsequent reasoning about time, but the reverse is not true (Boroditsky, 2000). Moreover, people's judgments about time are affected by simultaneous but irrelevant information about space, while the reverse again is not true (Casasanto and Boroditsky, 2008). Since perceiving and thinking about space affects perception and reasoning about time, we can infer that temporal cognition re-uses aspects of spatial cognition.

But time does not in fact have spatial extent. Thus, though languages systematically cast it in terms of one-dimensional space, they are unconstrained in terms of which axis it should be mapped onto. As a result, people in different cultures talk and also appear to think about time by mapping it onto different spatial axes. Mandarin Chinese describes time not only horizontally but also vertically, such that the past is above and the future below (Boroditsky, 2001), and Aymara places the future behind and the past ahead (Núñez and Sweetser, 2006). These linguistic differences correlate with other measurable cognitive differences, as shown in behavioral priming tasks (Boroditsky, 2001) and bodily gestures during speech (Núñez and Sweetser, 2006).

This cross-linguistic and cross-cultural variation leads us to ask: what determines the axis people use when they map time onto space? Is it arbitrary - a product of historical accident? Or, the hypothesis pursued in the experimental work described below, do cultural conventions for interacting with space through time - conventions that are irrelevant to the concept of time itselfnonetheless affect how people map time to space? In other words, if you happen to be used to doing things where you start on the left and end on the right, or start at the top and end at the bottom, do you tend to think of time as moving in that same direction? The particular cultural convention we will be looking at is the how people use space when reading and writing in their native language. This question is important from the broader perspective of how culture-specific ways of interacting with the world affect individual cognition.

WRITING DIRECTION MIGHT INFLUENCE THE SPACE TO TIME MAPPING Reading and writing are among the most frequent and most spatially systematic ways that literate people interact with the world. During reading and writing, we orient our eyes (and in some cases, our hands) to a location dictated by our writing system. Writing systems vary in the direction in which the text is written. Knowing how to read and write a particular language thus entails mastery 
of perceptual and motor routines whose particular spatial characteristics are determined by the conventional orientation of the writing system. To write in English, one starts with the first word at the top left and moves rightward and then downward, while a Taiwanese speaker of Chinese typically starts on the top right and moves downward then leftward. Similarly, reading in the two languages requires readers to begin collecting visual information at the appropriate, different, parts of the visual field, and then move gaze appropriately.

But does the conventional orientation of writing systems affect how people interact and think with space beyond reading and writing? More specifically, does writing system orientation influence our spatial representations of arbitrary sequences of events that are themselves not intrinsically spatially arrayed?

There is some evidence suggesting that writing system orientation may influence aspects of cognition other than writing. For example, in speakers of some European languages, like English and French, which are written from left to right (LR), the mental representation of numerical magnitude is related to the left-right axis. Large numbers elicit faster rightwards responses, and small numbers faster leftward responses (the SNARC effect - Dehaene et al., 1993). But Arabic speakers (Arabic is written from right to left, RL) display a spatially reversed SNARC effect, in which larger numbers are accessed faster on the left (Shaki et al., 2009). This effect only obtains with literate Arabic speakers (Zebian, 2005), which suggests a causal relation between practice reading and writing in a particular direction and the direction of the mental number line. While the number line is obviously distinct from temporal order, this result suggests that writing direction can have effects on arbitrarily spatially arrayed linear concepts.

What's more, there is good reason to believe that writing system direction affects not merely the mental number line, but also the mental representation of sequences in general. For instance, Gevers et al. (2003) have found a SNARC-like effect not with numbers but with non-numerical ordinal information. People speaking languages with different writing direction might thus also represent sequences differently. This result it merely suggestive, since it does not contrast populations who use different writing systems, but it does invite the possibility that the spatial depiction of ordinal information could be affected by writing system.

More compelling evidence that writing direction affects temporal cognition comes from work by Tversky et al. (1991), who asked English, Hebrew, and Arabic speakers to place stickers corresponding to temporally ordered events (like breakfast, lunch, and dinner) on a surface. English speakers showed a strong tendency to align them from LR, while Arabic speakers tended to align them from RL. Hebrew speakers showed a mixed response pattern.

However, these findings do not compel us to conclude that it is the writing system direction differences that are responsible for the different preferences in representing time spatially. English, Hebrew, and Arabic are spoken by populations that differ markedly. These different populations might use different metaphorical construals of time as space, which affect their spatial representations of time. In addition, these populations differ along other cultural dimensions; differences in how calendars, holy books, or other artifacts are constructed, among a host of other cultural differences, could effect differences in spatial construals of time. To eliminate these potential confounds, it would be preferable to find populations of speakers who are as closely matched as possible in that they share history, cultural practices, and language, while differing to the extent possible only in the direction of their writing systems.

In the pages below, we describe experimental work in a similar vein to Tversky et al.'s (1991) that investigates potential effects of writing system direction on spatial construals of time. Our work makes a novel contribution by looking at two closely matched populations - native speakers of Mandarin Chinese from both Mainland China and Taiwan - who, despite speaking the same language, write in different directions. The results suggest that when language and many aspects of culture are controlled for, writing direction can still affect how people map space onto time. If this is correct, then it is an example of how culture-specific aspects of how people interact with the world become internalized such that they affect other mental operations.

\section{MATERIALS AND METHODS}

The experimental design was quite simple. We used an arrangement task, in which participants were asked to spatially arrange cards printed with pictures depicting three stages of development of a natural entity, like a plant or a human, from the earliest to the latest stage. The purpose was to examine whether participants from different populations arrange sequences in different directions. Hypothetically, conventional writing orientation might affect the orientation of sequential information, so native speakers of different languages, written in different directions, might tend to arrange the images differently, and in alignment with their native writing and reading direction.

We included participants from three populations. First, we used people from Taiwan. In Taiwan, Standard Mandarin is the official state language and the language of instruction in schools. Writing in Taiwan at the time when data was collected, in 2004, was predominantly in the traditional Chinese style - in top to bottom (TB) columns, arranged from RL (Figure 1), though it was also written LR. (In subsequent years, the balance of writing in Taiwan has shifted farther in the LR direction, including writing on the web as well as in government documents.) We contrasted the performance of participants sampled from this population

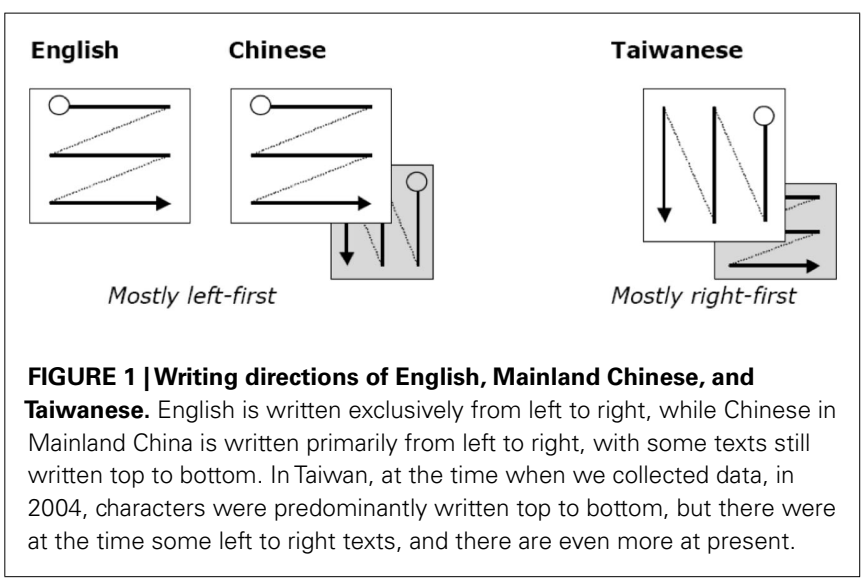


with those of participants from Mainland China. In Mainland China, as in Taiwan, Standard Mandarin is the official language, but text is predominantly written LR, though it can also, less frequently, be written TB. (We will intermittently refer to Mainland Chinese participants as Chinese in this paper, contrasted with Taiwanese). These two populations - Chinese and Taiwanese - serve as a promising contrast case, since they share a language as well as a great deal of culture and social history (the two countries were politically separated only in the twentieth century, and Taiwan's de facto independence is not recognized by Mainland China).

To augment this contrast pair, we also included American native English speakers, in the interest of determining the extent to which our findings replicate those reported in previous work (e.g., Tversky et al., 1991). English is of course written LR, like Chinese in Mainland China, and as a result, we expect English speakers to behave more like the Mainland Chinese than like the Taiwanese participants.

The task we used was entirely non-linguistic, designed as such for two reasons. First, as Tversky et al. (1991) argue "many pictorial communiqués are produced similarly by and can be comprehended by speakers of different languages with little or no training" (p. 516). So, pictorial stimuli can minimize any unnecessary bias provoked by linguistic codes (like numbers or words) on speakers of different languages. Second, our main interest is in the relationship between the direction of different writing systems (language) and the representation of time in the absence of reading or writing.

\section{PARTICIPANTS}

Ten right-handed English speakers, aged between 20 and 50 years, 33 right-handed Chinese participants, aged between 23 and 45 years ( mean $=24.6$ years), and 38 right-handed Taiwanese participants aged between 20 and 49 years (mean $=25.3$ years) were each tested individually. All English speakers were monolinguals, except for three who reportedly spoke some Spanish. (Spanish is written LR, like English, so these participants were not excluded.) All the Taiwanese and Chinese participants were native speakers of Standard Mandarin and were English L2 speakers. They were all born in Taiwan or Mainland China, respectively, and received education there before leaving for the United States between 0.2 and 6 years before being tested. Mean length of time that Taiwanese and Chinese participants had been in the United States was statistically indistinguishable. All additionally claimed that they still read Chinese occasionally even though they were now in the United States.

All participants in all three groups were either doing or had already finished a Bachelor's degree or equivalent, thus having reached a relatively high level of literacy.

\section{MATERIALS}

The materials were composed of five sets of black-and-white images. Each set contained three pictures depicting a growing process of a living thing. The five sets of pictures were:

(1) seed - sapling - tree

(2) egg - chick - chicken

(3) larva - pupa - butterfly

(4) tadpole - froglet - adult frog

(5) baby - girl - woman
Each picture was printed on a 3 " diameter round piece of white paper. Another, larger round piece of white cardboard with a 9.1" diameter was prepared as the tray for participants to arrange the small paper circles on. We used round cards on a round surface to minimize any similarity between this task and typical features of writing and reading.

\section{PROCEDURE}

Participants were asked to arrange each set of three pictures in sequence from the earliest to the latest stage on the cardboard, and were limited to $8 \mathrm{~s}$. All instructions were presented orally in English (for the English-speaking participants) or Standard Mandarin (for the Chinese and Taiwanese participants). No written materials were provided, so as to avoid the possibility of priming from reading.

After being provided with instructions, the larger cardboard circle was placed in front of participants. Participants were then handed the first set of three randomly ordered pictures in a stack, face-down. Participants flipped them over at the same time and arranged them in a sequential order.

Each subsequent set of pictures was presented separately and was analyzed individually. After the participant had completed each set, the experimenter coded the spatial arrangement. After completion of the five sets, participants were asked to explain why they thought they had arranged the cards as they had, in a brief post-experiment interview. Including instruction, the experiment took $5 \mathrm{~min}$ on average.

We predicted that English-speaking participants should tend to arrange pictures LR, following the direction of their writing system, that Mainland Chinese participants should do the same, and that Taiwanese participants should show a stronger tendency to arrange pictures $\mathrm{TB}$, in accordance with the predominance of this direction in their writing and reading experience.

\section{RESULTS \\ CODING}

The arrangements were straightforward to score and no data were missing. Participants displayed five arrangement patterns: LR, RL, $\mathrm{TB}$, bottom to top (BT), and clockwise (CW) starting from the top. Examples of each are in Figure 2. Directions are defined from the perspective of the participant, so TB actually involved arranging the three cards with the temporally earliest one farthest away from the participant along the mid-sagittal axis, and BT placed the temporally earliest card closest to the participant along the same axis.

All but three participants used exactly the same orientation for each of the five sets of pictures (s)he ordered. In those three cases where the orientation differed across a participant's responses, we counted the participant's response pattern for the purpose of statistical analysis as the one (s)he used the majority of the time.

\section{PATTERNS OF RESPONSE BY NATIVE LANGUAGE}

As seen in Table 1, below, the English speakers only used the LR arrangement pattern. Participants from Mainland China displayed a strong tendency to adopt the same LR arrangement pattern, though a few also used a TB orientation. For the Taiwanese participants, all five patterns were observed, with the largest numbers 


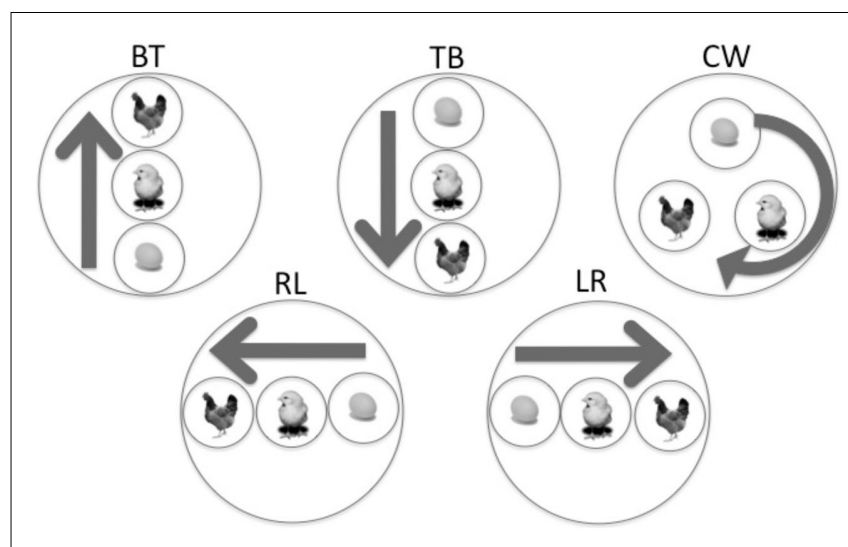

FIGURE 2 |The five observed patterns of arrangement.

Table 1 | Arrangement direction frequencies by group.

\begin{tabular}{lcccr}
\hline Direction & English & Chinese & Taiwanese & Total \\
\hline LR & 10 & 26 & 13 & 49 \\
RL & 0 & 0 & 7 & 7 \\
TB & 0 & 5 & 13 & 18 \\
BT & 0 & 1 & 2 & 3 \\
CW & 0 & 1 & 3 & 4 \\
Total & 10 & 33 & 38 & 81 \\
\hline
\end{tabular}

being the LR and TB orientations. By contrast with the English and Chinese participants, who never placed the images in a RL orientation, the Taiwanese participants did so about $20 \%$ of the time.

The critical expected differences were for English and Chinese participants to have proportionally more responses in the LR pattern than Taiwanese participants, who are anticipated to have relatively more TB. Pairwise chi-square tests comparing these two critical conditions reveal significant differences between English and Taiwanese $\left(\chi^{2}=7.83, p=0.005\right.$; Yates' $\left.\chi^{2}=5.81, p=0.02\right)$ and Chinese and Taiwanese $\left(\chi^{2}=7.51, p=0.006\right.$; Yates' $\chi^{2}=6.02$, $p<0.01$ ), but not between English and Chinese (n.s.). There were also RL responses in the Taiwanese data but none produced by the other groups. Comparing LR with RL responses again revealed significant differences between English and Taiwanese $\left(\chi^{2}=4.57\right.$, $p=0.03$; Yates' $\left.\chi^{2}=2.82, p=0.09\right)$ and Chinese and Taiwanese $\left(\chi^{2}=10.73, p=0.001\right.$; Yates' $\left.\chi^{2}=8.19, p=0.004\right)$ but not English and Chinese (n.s.). As predicted, English and Chinese participants have different preferences for arranging sequential information than the Taiwanese participants do.

\section{DISCUSSION}

\section{PATTERNS OF RESPONSE BY NATIVE LANGUAGE}

English-speaking participants, as expected, arranged pictures LR. For the most part, so did Chinese participants. For both, their spatial depictions of time were consistent with the dominant LR pattern present in their writing and reading experience. But there was a bit more variability among the Chinese participants than the English speaking ones. Five participants used a TB arrangement pattern. One explanation for these results is continuing cultural presence of the TB writing system in old texts that predate the shift to LR in the 1950s, or on other artifacts, like calligraphy and signage on government buildings.

The results from the Taiwanese participants were more variable still. Responses in the post-test interview may help us understand the broad range of responses the experiment elicited. We asked each participant why (s)he arranged the pictures in the particular pattern we observed. For LR and TB patterns, the answers were predictable. Participants, when asked to reflect on their behavior, reported arranging these pictures mainly based on their reading and writing habits. As Mandarin was written TB or LR in Taiwan at the time of data collection, heterogeneous results are not surprising. And as all participants were residing in the United States at the time of data collection, it is possible that the Chinese and Taiwanese speakers were more likely to use LR due to exposure to English writing. (However, when we did a median split of Chinese and Taiwanese participants based on the length of time they had been residing in the United States, we found no significant difference between the two halves.) The RL result may relate to the secondary direction of standard writing in Taiwan; while it is primarily written from $\mathrm{TB}$, each column is placed to the left of the preceding one. Other response types (BT and CW, for example), elicited responses not specific to writing. Some BT participants explained that growing things go from BT, while some CW participants evoked the cyclicity of growth and reproduction.

Aside from writing direction, there are also a few linguistic features that distinguish the Standard Mandarin spoken in Mainland China and Taiwan, including lexical differences, and some of these might in principle be responsible for the difference in behavior we found. We cannot conclusively rule out all differences as potential factors, but we can look at the most relevant possible difference, which would be metaphorical language for time. If Taiwanese speakers use a preponderance of vertical language for time, while Mainland Chinese speakers use relatively little vertical metaphorical time language, then this possible confound could explain the Taiwanese tendency to represent time TB. However, corpus research shows that in fact Taiwanese speakers use relatively little vertical time language, about half as much as horizontal metaphorical time language (Chen, 2007), which matches or may even be less frequent than vertical time language in Mainland China (Rong, 2007). So differences in how time is construed metaphorically are unlikely to account for the difference in responses we observed; they would in fact predict the opposite effect if anything. However, the existence of vertical time language in both dialects might help to explain why a small portion of Chinese participants placed the earliest picture at the top and the latest at the bottom, while no English participants did so.

\section{IMPLICATIONS AND FURTHER DIRECTIONS}

The direction of a writing system affects production of sequential arrangements. For English participants, the exceptionless LR pattern demonstrates that spatial representations for sequences take left as the beginning, proceeding toward the right, while this tendency is slightly less strong among Mainland Chinese participants. For Taiwanese participants, the varying patterns, as discussed above, tell us that while the writing system may be the most 
important factor influencing people's representation of sequences, it can not be the only one. On the assumption that there are no innate biological differences distinguishing the populations with respect to their preferred spatializations of time, there must be differences in the experiences members of these different populations have that lead to the differences in behavior. These other factors may include differences in cultural values and practices.

Though there are many other possible factors, writing system appears to influence people's use of space. Since Mainland China and Taiwan share the same language, many core cultural values, traditions, and much of their history, if it were any of these cultural factors other than writing direction that were causing differences between English and Taiwanese participants, Chinese participants should pattern with Taiwanese participants. Yet, as we have seen, the behavior of the Chinese participants is closely aligned with that of the English participants and different from that of the Taiwanese participants.

Despite the similarity of Taiwanese and Mainland Chinese culture, it might still be that other cultural factors, and not just writing system orientation, are responsible for the effects reported above. In order to further understand exactly what the causes of these cross-linguistic differences are, the same experiments might be conducted with prelinguistic children or illiterate adults, who would have less experience with writing systems, and thus would be less influenced by them. If it is truly writing orientation that is the major factor in the results described above, then the effect of native language should disappear with such participants. Similarly, reader/writers of unrelated languages with the various writing orientations, like Arabic, Japanese, and Korean, could provide useful points of comparison. Another way to pursue this line of research further would be to experimentally introduce experience with a new writing system to participants drawn from a single population, to see whether - over time - such a manipulation could affect their spatial representation of time.

\section{REFERENCES}

Boroditsky, L. (2000). Metaphoric structuring: understanding time through spatial metaphors. Cognition $75,1-28$.

Boroditsky, L. (2001). Does language shape thought? English and Mandarin speakers' conceptions of time. Cogn. Psychol. 43, 1-22.

Casasanto, D., and Boroditsky, L. (2008). Time in the mind: using space to think about time. Cognition 106, 579-593.

Chen, J. Y. (2007). Do Chinese and English speakers think about time differently? Failure of replicating Boroditsky (2001). Cognition 104, 427-436.

Clark, H. (1973). "Space, time semantics, and the child," in Cognitive Development and the Acquisition of Language, ed. T. E. Moore (New York: Academic Press), 27-63.

Dehaene, S., Bossimi, S., and Giraux, P. (1993). The mental representation

It is also possible that writing direction has effects on other cognitive operations than the representation of time. Space is used as a basis for a variety of abstract concepts, like power, morality, happiness, and so on (Lakoff and Johnson, 1980). We leave it open to future work whether time is the only abstract concept to be construed differently depending on a culture's writing system direction.

Finally, it is worth noting that the method we used did not distinguish between the representation of sequence and the representation of time per se. It is possible that the effect we observed was the result of spatializations of sequence and not time - in that case, we might expect to find similar effects with arranging atemporal sequences, like colors, for example. The current design leaves open the question of which of these facilities we are tapping into.

\section{CONCLUSION}

To conclude, these results support the hypothesis that writing system orientation influences spatial cognition. We have seen that the location where a writing system starts is where people spatially represent the beginnings of temporal sequences. These differences in behavior may in turn influence how we interpret the world and language about it. More broadly, it seems that writing system orientation is an idiosyncratic linguistic characteristic that can have an impact on our cognitive system in general, like other linguistic features that have relativistic effects. The details of a language - in this case an apparently superficial feature of how people in a given culture interact with its written form, seems to shape the way that people think about something totally unrelated.

As Griffin (2004) argues, "eye movements are tied to our organization of information." Patterns of interaction with writing appear to seep out beyond the borders of language. Learning to use a writing system creates routines of interaction with space that affect how we map time onto it.

Spatial-Temporal Metaphor. Foreign Language Research 2.

Shaki, S., Fischer, M. H., and Petrusic, W. M. (2009). Reading habits for both words and numbers contribute to the SNARC effect. Psychon. Bull. Rev. $16,328-331$.

Traugott, E. (1978). “On the expression of spatiotemporal relations in language," in Universals of Human Language: Word Structure, Vol. 3, ed. J. H. Greenberg (Stanford, CA: Stanford University Press), 369-400.

Tversky, B., Sol, K., and Winter, A. (1991). Cross-cultural and developmental trends in graphic productions. Cogn. Psychol. 23, 515-557.

Zebian, S. (2005). Linkages between number concepts, spatial thinking, and directionality of writing: the SNARC effect and the reverse SNARC effect in English and Arabic monoliterates, biliterates and illiterate Arabic speakers. J. Cogn. Cult. 5, 165-191.
Conflict of Interest Statement: The authors declare that the research was conducted in the absence of any commercial or financial relationships that could be construed as a potential conflict of interest.

Received: 10 January 2012; accepted: 26 March 2012; published online: 10 April 2012.

Citation: Bergen BK and Chan Lau TT (2012) Writing direction affects how people map space onto time. Front. Psychology 3:109. doi: 10.3389/fpsyg.2012.00109 This article was submitted to Frontiers in Cultural Psychology, a specialty of Frontiers in Psychology.

Copyright (C) 2012 Bergen and Chan Lau. This is an open-access article distributed under the terms of the Creative Commons Attribution Non Commercial License, which permits non-commercial use, distribution, and reproduction in other forums, provided the original authors and source are credited. 